\title{
The impacts of co-infection of HIV and Falciparum malaria on cellular immune responses of pregnant mothers and their babies' post-delivery
}

\begin{abstract}
It has been postulated that co-infection of HIV and malaria complicate diagnosis and treatment of either infection or both. Other studies indicated that clinical malaria led to HIV RNA level rise, and decline as much as 40 cells/ $\mu \mathrm{L} /$ year with each episode of malaria. This study assessed the impacts of on the number of $\mathrm{CD}^{+}$cells and levels of cytokines produced in pregnant women and their babies post-delivery. Saki; (Latitudes $8^{\circ} 26^{\prime}$ and $9^{\circ} 5^{\prime}$ North and Longitudes $2^{\circ} 45^{\prime}$ and $3^{\circ} 37^{\prime}$ East) known for its seroprevalence and co-infection in Oyo State, Nigeria. 149 pregnant mothers screened of HIV and P. Falciparum after due informed consent and ethical approval. Thirty babies born to sero-positive mothers were enrolled and followed-up post-partum. Blood samples $(5 \mathrm{~mL})$ collected were screened for the presence of malaria parasites. Data obtained were analyzed using Student t-test and ANOVA. Co-infection impacted on the weight of babies and making them susceptible to be anemic. The co-infected had higher parasitemia compared to their mono and uninfected counterparts. Co-infected mothers had lower CD4+ lymphocytes. The high level in TNF- $\alpha$ could favour viral replication, vertical transmission of HIV and indicative of compromised cellular immunity. IL-2 suggested possible placental malaria. In consonance with earlier studies; IL-10 played Immuno-mediatory role. IFN- $\gamma$ levels suggest increased chances of poor birth outcomes. The co-infection rate impacted on the percentage of CD4 lymphocytes and modulated the plasma levels of cytokines.
\end{abstract}

Keywords: sero prevalence, episode of malaria, cytokines, impacted
Volume I Issue $3-2017$

\author{
Adeoti O Micheal \\ Department of Science Laboratory Technology, University of \\ Ibadan, Nigeria
}

Correspondence: Adeoti O Micheal, Department of Science Laboratory Technology, Cellular Parasitology Unit, University of Ibadan, Oke-Ogun Polytechnic Saki, Nigeria, Email txy23m@yahoo.com

Received: September 20, 2017 | Published: October 24, 2017
Abbreviations: CD4, clonal differentiation; HIV-human immunodeficiency virus; RNA, ribonucleic acid; IL, interleukin; IFN, interferon; ANOVA, analysis of variance

\section{Introduction}

It has been postulated that co-infection of HIV and malaria complicate diagnosis and treatment of either infection; thereby threatens the protective cellular barrier mounted to establish pregnancy as well as maintain the well-being of the foetus. Other studies indicated that clinical malaria led to HIV RNA level rise, and decline as much as 40 cells $/ \mu \mathrm{L} /$ year with each episode of malaria. This study examined the impacts of $P$. Falciparum and HIV co-infection on the number of $\mathrm{CD} 4^{+}$cells and levels of cytokines produced in pregnant women and their babies post-delivery. ${ }^{1-2}$

\section{Methods}

Saki; (Latitudes $8^{\circ} 26^{\prime}$ and $9^{\circ} 5^{\prime}$ North and Longitudes $2^{\circ} 45^{\prime}$ and $3^{\circ}$ $37^{\prime}$ East) known for its seroprevalence and co-infection in Oyo State,
Nigeria. 149 pregnant mothers screened of HIV and P. Falciparum after due informed consent and ethical approval by UI/UCH Ethical Committee and 30 babies born to sero-positive mothers were enrolled and followed-up Post-partum. Blood samples $(5 \mathrm{~mL})$ collected were screened for the presence of malaria parasites. Patients' CD4 ${ }^{+}$ counts and plasma concentration of (TNF- $\alpha$, IL-2, IL-10 and IFN- $\gamma$ ) were determined by FACS and ELISA techniques respectively Data obtained were analyzed using Student t-test and ANOVA.

\section{Results}

Co-infection impacted on the weight of babies and likely anemic (Figure 1). The co-infected (Table 1) had higher parasitemia compared to their mono and uninfected counterparts (Figure 2A) (Figure 2B). Co-infected mothers had lower CD4+ lymphocytes (Figure 3). The high level in TNF- $\alpha$ could favour viral replication, vertical transmission of HIV and indicative of compromised cellular immunity (Figure 4). IL-2 suggested possible placental malaria (5B). In consonance with earlier studies; IL-10 played Immuno-mediatory role IFN- $\gamma$ levels suggest increased chances of poor birth outcomes (Figure 5).

Table I Participants by infection status

\begin{tabular}{llllll}
\hline Infection & Co-infected & HIV only & Malaria only & No-infection & Total \\
\hline Mothers & $34(22.8)$ & $1 \mathrm{I}(36.7)$ & $51(49.0)$ & $53(51.0)$ & $149(100.0)$ \\
Infants & $5(26.3)$ & $14(73.7)$ & $6(54.5)$ & $5(45.5)$ & $30(100.0)$ \\
Total & $39(21.8)$ & $25(13.9)$ & $57(31.8)$ & $58(32.5)$ & $179(100.0)$ \\
\hline
\end{tabular}




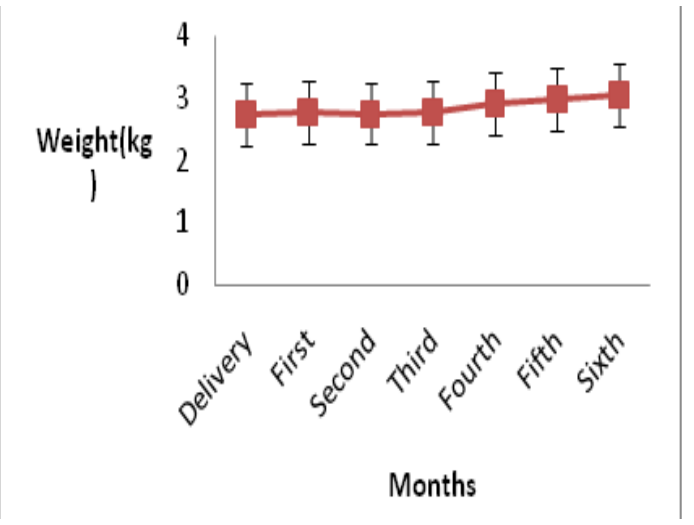

Figure I The mean weight of co-infected babies at delivery and early postdelivery.

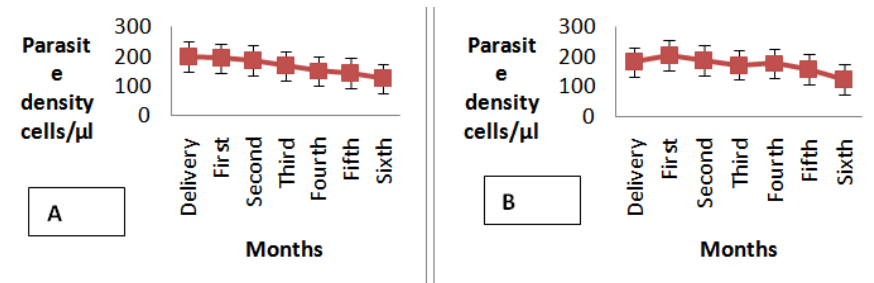

Figure $2 \mathrm{~A}$, The mean parasite density in babies post-delivery; B, The mean parasite density in co-infected babies.

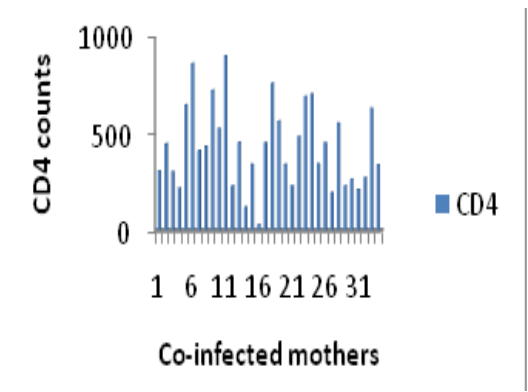

Figure $3 \mathrm{CD} 4$ count inco-infected mothers.

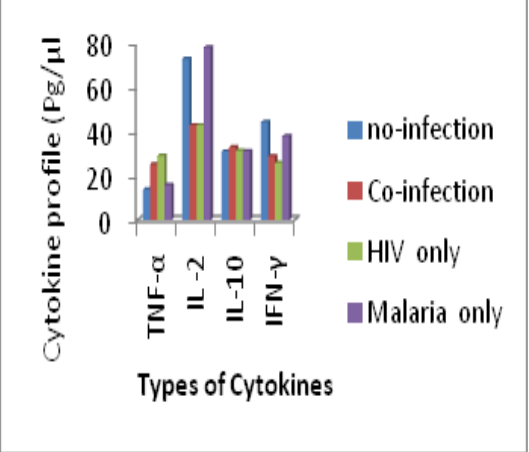

Figure 4 Cytokines in co-infected mothers.

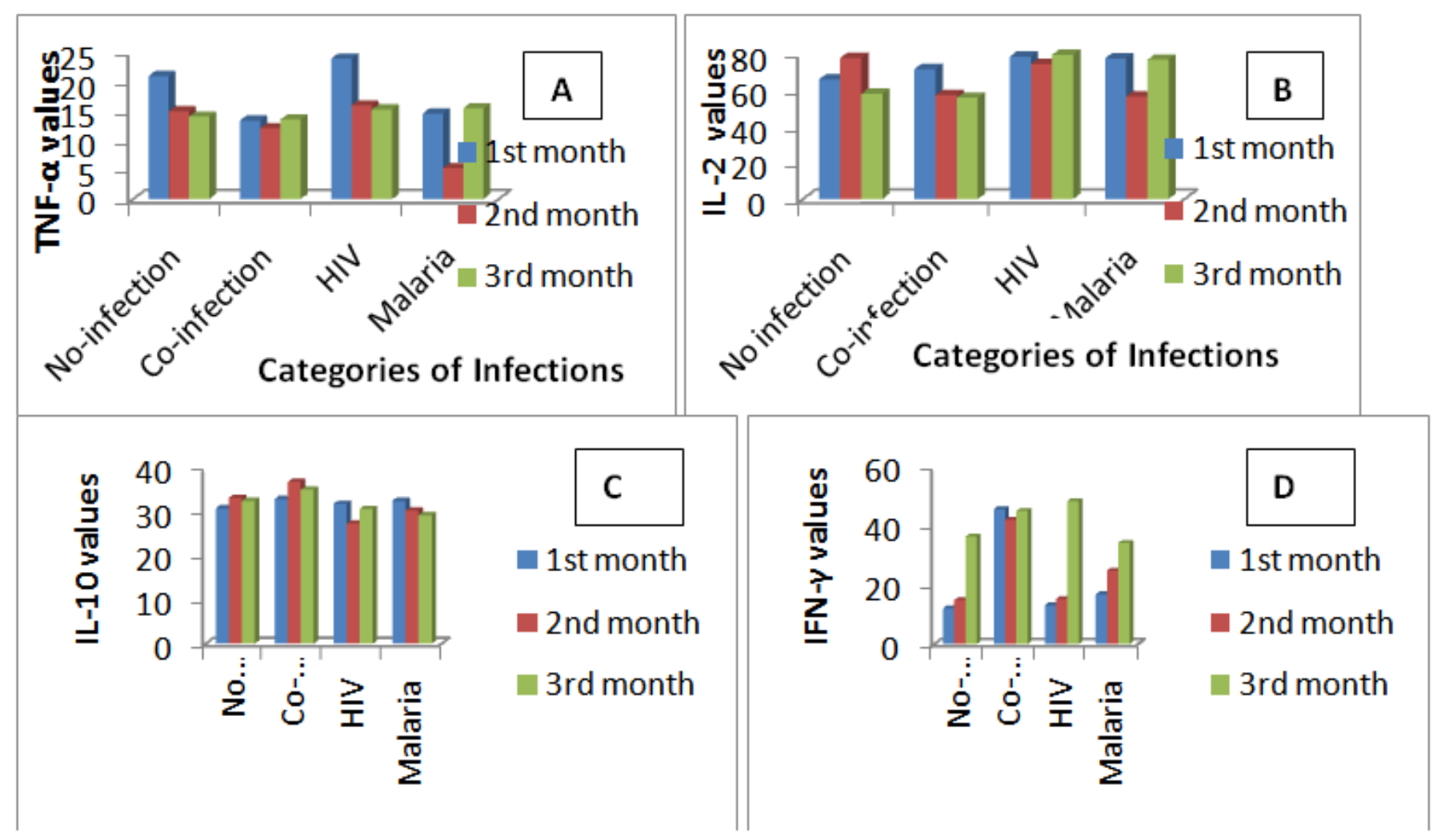

Figure 5 Cytokine levels in co-infected babies. 


\section{Conclusion}

The co-infection rate impacted on the percentage of CD4 lymphocytes and modulated the plasma levels of cytokines.

\section{Acknowledgements}

We acknowledge the contributions of colleagues, the participants and the authorities of the selected hospitals.

\section{Conflict of interest}

The Author declared that there is no conflict of interest.

\section{References}

1. Corbett EL, Steketee RW, TerKuile, et al. HIV-1/AIDS and the control of other infectious diseases in Africa. Lancet. 2002;359(9324):2177-2187.

2. World Health Organization. Malaria and HIV interactions and their implications for public health policy. World Health Organization. Geneva, Switzerland; 2011. 